\title{
Fitting Sentence Level Translation Evaluation with Many Dense Features
}

\author{
Miloš Stanojević and Khalil Sima'an \\ Institute for Logic, Language and Computation \\ University of Amsterdam \\ Science Park 107, 1098 XG Amsterdam, The Netherlands \\ \{m.stanojevic,k.simaan\}@uva.nl
}

\begin{abstract}
Sentence level evaluation in MT has turned out far more difficult than corpus level evaluation. Existing sentence level metrics employ a limited set of features, most of which are rather sparse at the sentence level, and their intricate models are rarely trained for ranking. This paper presents a simple linear model exploiting 33 relatively dense features, some of which are novel while others are known but seldom used, and train it under the learning-to-rank framework. We evaluate our metric on the standard WMT12 data showing that it outperforms the strong baseline METEOR. We also analyze the contribution of individual features and the choice of training data, language-pair vs. target-language data, providing new insights into this task.
\end{abstract}

\section{Introduction}

Evaluating machine translation (MT) output at the sentence/ segment level has turned out far more challenging than corpus/ system level. Yet, sentence level evaluation can be useful because it allows fast, finegrained analysis of system performance on individual sentences.

It is instructive to contrast two widely used metrics, METEOR (Michael Denkowski and Alon Lavie, 2014) and BLEU (Papineni et al., 2002), on sentence level evaluation. METEOR constantly shows better correlation with human ranking than BLEU (Papineni et al., 2002). Arguably, this shows that sentence level evaluation demands finer grained and trainable models over less sparse features. Ngrams, the core of BLEU, are sparse at the sentence level, and a mismatch for longer ngrams implies that BLEU falls back on shorter ngrams. In contrast, METEOR has a trainable model and incorporates a small, yet wider set of features that are less sparse than ngrams. We think that METEOR's features and its training approach only suggest that sentence level evaluation should be treated as a modelling challenge. This calls for questions such as what model, what features and what training objective are better suited for modelling sentence level evaluation.

We start out by explicitly formulating sentence level evaluation as the problem of ranking a set of compet- ing hypothesis. Given data consisting of human ranked system outputs, the problem then is to formulate an easy to train model for ranking. One particular existing approach (Ye et al., 2007) looks especially attractive because we think it meshes well with a range of effective techniques for learning-to-rank ( $\mathrm{Li}, 2011)$.

We deliberately select a linear modelling approach inspired by RankSVM (Herbrich et al., 1999), which is easily trainable for ranking and allows analysis of the individual contributions of features. Besides presenting a new metric and a set of known, but also a set of novel features, we target three questions of interest to the MT community:

- What kind of features are more helpful for sentence level evaluation?

- How does a simple linear model trained for ranking compare to the well-developed metric METEOR on sentence level evaluation?

- Should we train the model for each language pair separately or for a target language?

Our new metric dubbed BEER ${ }^{1}$ outperforms METEOR on WMT12 data showing the effectiveness of dense features in a learning-to-rank framework. The metric and the code are available as free software ${ }^{2}$.

\section{Model}

Our model is a linear combination of features trained for ranking similar to RankSVM (Herbrich et al., 1999) or, to readers familiar with SMT system tuning, to PRO tuning (Hopkins and May, 2011):

$$
\operatorname{score}(\text { sys })=\overrightarrow{\boldsymbol{w}} \cdot \overrightarrow{\boldsymbol{x}}_{\text {sys }}
$$

where $\overrightarrow{\boldsymbol{w}}$ represents a weight vector and $\overrightarrow{\boldsymbol{x}}_{\text {sys }}$ a vector of feature values for system output sys. Looking at evaluation as a ranking problem, we contrast (at least) two system translations good and bad for the same source sentence. Assuming that humanRank $($ good $)>$ humanRank $($ bad $)$ as ranked

\footnotetext{
${ }^{1}$ BEER participated on WMT14 evaluation metrics task where it was the highest scoring sentence level evaluation metric on average over all language pairs (Stanojević and Sima'an, 2014)

${ }^{2}$ https://github.com/stanojevic/beer
} 
by human judgement, we expect metric score $(\cdot)$ to fulfill score $($ good $)>\operatorname{score}($ bad $)$ :

$$
\begin{array}{rr}
\overrightarrow{\boldsymbol{w}} \cdot \overrightarrow{\boldsymbol{x}}_{\text {good }}>\overrightarrow{\boldsymbol{w}} \cdot \overrightarrow{\boldsymbol{x}}_{\text {bad }} & \Leftrightarrow \\
\overrightarrow{\boldsymbol{w}} \cdot \overrightarrow{\boldsymbol{x}}_{\text {good }}-\overrightarrow{\boldsymbol{w}} \cdot \overrightarrow{\boldsymbol{x}}_{\text {bad }}>0 & \Leftrightarrow \\
\overrightarrow{\boldsymbol{w}} \cdot\left(\overrightarrow{\boldsymbol{x}}_{\text {good }}-\overrightarrow{\boldsymbol{x}}_{\text {bad }}\right)>0 & \wedge \\
\overrightarrow{\boldsymbol{w}} \cdot\left(\overrightarrow{\boldsymbol{x}}_{\text {bad }}-\overrightarrow{\boldsymbol{x}}_{\text {good }}\right)<0 &
\end{array}
$$

The two feature vectors $\left(\overrightarrow{\boldsymbol{x}}_{\text {good }}-\overrightarrow{\boldsymbol{x}}_{b a d}\right)$ and $\left(\overrightarrow{\boldsymbol{x}}_{b a d}-\right.$ $\left.\overrightarrow{\boldsymbol{x}}_{\text {good }}\right)$ can be considered as positive and negative instances for training our linear classifier. For training this model, we use Logistic Regression from the Weka toolkit (Hall et al., 2009).

\section{Features}

Generally speaking we identify adequacy and fluency features. For both types we devise far less sparse features than word ngrams.

Adequacy features We use precision $P$, recall $R$ and F1-score $F$ as follows:

$P_{\text {func }}, R_{\text {func }}, F_{\text {func }}$ on matched function words

$P_{\text {cont }}, R_{\text {cont }}, F_{\text {cont }}$ on matched content words

$P_{\text {all }}, R_{\text {all }}, F_{\text {all }}$ on matched words of any type

$P_{\text {char }}, R_{\text {char }}, F_{\text {char }}$ matching of the char ngrams

By differentiating between function and non-function words, our metric weighs each kind of words according to importance for evaluation. Matching character ngrams, originally proposed in (Yang et al., 2013), rewards certain translations even if they did not get the morphology completely right. Existing metrics use stemmers for this, but using character ngrams is independent of the availability of a good quality stemmer. Higher-order character ngrams have less risk of sparse counts than word ngrams. In our experiments we used char ngrams for $n$ up to 6 , which makes the total number of adequacy features 27 .

Fluency features To evaluate word order we follow (Isozaki et al., 2010; Birch and Osborne, 2010) in representing reordering as a permutation $\pi$ over $[1 . . n]$ and then measuring the distance to the ideal monotone permutation $\langle 1,2, \cdots, n\rangle$. We present a novel approach based on factorization into permutation trees (PETs) (Zhang and Gildea, 2007), and contrast it with Kendall $\tau$ (Birch and Osborne, 2010; Isozaki et al., 2010). PETs are factorizations of permutations, which allows for an abstract and less sparse view of word order as exemplified next. Kendall score was regularly shown to have high correlation with human judgment on distant language pairs (Isozaki et al., 2010; Birch and Osborne, 2010).
Features based on PETs We informally review PETs in order to exploit them for novel ordering features. We refer the reader to (Zhang and Gildea, 2007) and (Maillette de Buy Wenniger and Sima'an, 2011) for a formal treatment of PETs and efficient factorization algorithms.

A PET of permutation $\pi$ is a tree organization of $\pi$ 's unique, atomic building blocks, called operators. Every operator on a PET node is an atomic permutation (not factorizing any further), ${ }^{3}$ and it stands for the permutation of the direct children of that node. Figure 1a shows an example PET that has one 4-branching node with operator $\langle 2,4,1,3\rangle$, two binary branching nodes of which one decorated with the inverted operator $\langle 2,1\rangle$ and another with the monotone $\langle 1,2\rangle$.

PETs have two important properties making them attractive for measuring order difference: firstly, order difference is measured on the operators - the atomic reordering building blocks of the permutation, and secondly, the operators on higher level nodes capture hidden ordering patterns that cannot be observed without factorization. Statistics over ordering patterns in PETs are far less sparse than word or character ngram statistics.

Intuitively, among the atomic permutations, the binary monotone operator $\langle 1,2\rangle$ signifies no ordering difference at all, whereas the binary inverted $\langle 2,1\rangle$ signifies the shortest unit of order difference. Operators of length four like $\langle 2,4,1,3\rangle$ (Wu, 1997) are presumably more complex than $\langle 2,1\rangle$, whereas operators longer than four signify even more complex order difference. Therefore, we devise possible branching feature functions over the operator length for the nodes in PETs:

- factor 2 - with two features: $\Delta_{[]}$and $\Delta_{<>}$(there are no nodes with factor $3(\mathrm{Wu}, 1997))$

- factor 4 - feature $\Delta_{=4}$

- factor bigger than 4 - feature $\Delta_{>4}$

Consider permutations $\langle 2,1,4,3\rangle$ and $\langle 4,3,2,1\rangle$, none of which has exactly matching ngrams beyond unigrams. Their PETs are in Figures $1 \mathrm{~b}$ and 1c. Intuitively, $\langle 2,1,4,3\rangle$ is somewhat less scrambled than $\langle 4,3,2,1\rangle$ because it has at least some position in correct order. These "abstract ngrams" pertaining to correct ordering of full phrases could be counted using $\Delta_{[]}$which would recognize that on top of the PET in $1 \mathrm{~b}$ there is a binary monotone node, unlike the PET in Figure 1c which has no monotone nodes at all.

Even though the set of operators that describe a permutation is unique for the given permutation, the ways in which operators are combined (the derivation tree) is not unique. For example, for the fully monotone

\footnotetext{
${ }^{3}$ For example $\langle 2,4,1,3\rangle$ is atomic whereas $\langle 4,3,2,1\rangle$ is not. The former does not contain any contiguous sub-ranges of integers whereas the latter contains sub-range $\{2,3,4\}$ in reverse order $\langle 4,3,2\rangle$, which factorizes into two binary inverting nodes $\mathrm{cf}$. Fig. $1 \mathrm{c}$.
} 


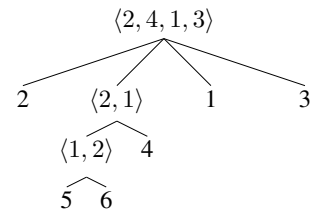

(a) Complex PET

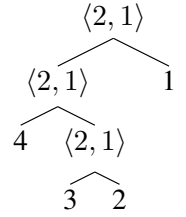

(d) Alternative fully inverted PET

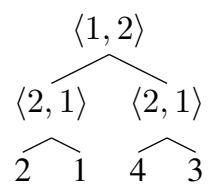

(b) PET with inversions

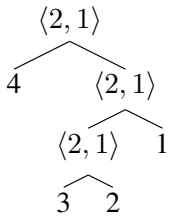

(f) Alternative fully inverted PET

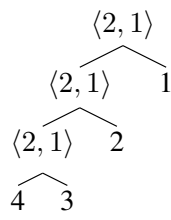

(c) Canonical fully inverted PET

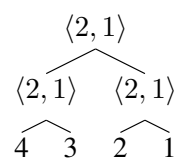

(e) Alternative fully inverted PET

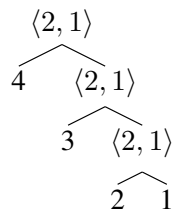

(g) Alternative fully inverted PET

Figure 1: Examples of PETs

permutation $\langle 4,3,2,1\rangle$ there are 5 possible derivations (PETs) presented in Figures 1c, 1d, 1e, 1f and 1g. The features on PETs that we described so far look at the operators independently (they treat a derivation as a set of operators) so differenct derivations do not influence the score-whichever derviation we use we will get the same feature score. However, the number of derivations might say something about the goodness of the permutation. Similar property of permutations was found to be helpful earlier in (Mylonakis and Sima'an, 2008) as an ITG prior for learning translation rule probabilities.

Permutations like $\langle 4,3,2,1\rangle$ and $\langle 2,4,3,1\rangle$ have the same set of operators, but the former factorizes into more PETs than the latter because $\langle 4,3\rangle$ must group first before grouping it with 1 and 2 in $\langle 2,4,3,1\rangle$. The "freedom to bracket" in different ways could be a signal of better grouping of words (even if they have inverted word order). Hence we exploit one more feature:

$\Delta_{\text {count }}$ the ratio between the number of alternative PETs for the given permutation, to the number of PETs that could be built if permutation was perfectly grouped (fully monotone or fully inverted).

Finding the number of PETs that could be built does not require building all PETs or encoding them in the chart. The number can be computed directly from the canonical left-branching PET. Since multiple different PETs appear only in cases when there is a sequence of more than one node that is either $\langle 1,2\rangle$ or $\langle 2,1\rangle$ (Zhang et al., 2008), we can use these sequences to predict the number of PETs that could be built. Let $X$ represent a set of sequences of the canonical derivation. The number of PETs is computed in the following way:

$$
\begin{gathered}
\# \text { PETs }=\prod_{x \in X} \operatorname{Cat}(|x|) \\
\operatorname{Cat}(n)=\frac{1}{n+1}\left(\begin{array}{c}
2 n \\
n
\end{array}\right)
\end{gathered}
$$

where $\operatorname{Cat}(\cdot)$ is a Catalan number. The proof of this formula is beyond the scope of this paper. The reader can consider the example of the PET in Figure 1c. That derivation has one sequence of monotone operators of length 3. So the number of PETs that could be built is $\operatorname{Cat}(3)=5$.

\section{Experiments}

We use human judgments from the WMT tasks: WMT13 is used for training whereas WMT12 for testing. The baseline is METEOR's latest version (Michael Denkowski and Alon Lavie, 2014), one of the best metrics on sentence level. To avoid contaminating the results with differences with METEOR due to resources, we use the same alignment, tokenization and lowercasing (-norm in METEOR) algorithms, and the same tables of function words, synonyms, paraphrases and stemmers.

Kendall $\tau$ correlation is borrowed from WMT12 (Callison-Burch et al., 2012):

$$
\tau=\frac{\# \text { concordant }-\# \text { discordant }-\# \text { ties }}{\# \text { concordant }+\# \text { discordant }+\# \text { ties }}
$$

\#concordant represents the number of pairs ordered in the same way by metric and by human, \#discordant the number of opposite orderings and \#ties the number of tied rankings by metric.

Beside testing our full metric BEER, we perform experiments where we remove one kind of the following features at a time:

1. char n-gram features (P, R and F-score)

2. all word features (P, R and F-score for all, function and content words),

3. all function and content words features

4. all F-scores (all words, function words, content words, char ngrams) 


\begin{tabular}{l|llllllll|l} 
metric & en-cs & en-fr & en-de & en-es & cs-en & fr-en & de-en & es-en & avg $\tau$ \\
\hline BEER without char features & 0.124 & 0.178 & 0.168 & 0.149 & 0.121 & 0.17 & 0.179 & 0.078 & 0.146 \\
BEER without all word features & 0.184 & 0.237 & 0.223 & 0.217 & 0.192 & 0.209 & 0.243 & 0.199 & 0.213 \\
BEER without all F-scores & 0.197 & 0.243 & 0.219 & 0.22 & 0.177 & 0.227 & 0.254 & 0.211 & 0.219 \\
METEOR & 0.156 & 0.252 & 0.173 & 0.202 & 0.208 & 0.249 & 0.273 & 0.246 & 0.22 \\
BEER without PET features & 0.202 & 0.248 & 0.243 & 0.225 & 0.198 & 0.249 & 0.268 & 0.234 & 0.233 \\
BEER without function words & 0.2 & 0.245 & 0.231 & 0.227 & 0.189 & 0.268 & 0.267 & 0.253 & 0.235 \\
BEER without fluency features & 0.201 & 0.248 & 0.236 & 0.223 & 0.202 & 0.257 & 0.283 & 0.243 & 0.237 \\
BEER without Kendall $\tau$ & 0.205 & 0.246 & 0.244 & 0.227 & 0.202 & 0.257 & 0.282 & 0.248 & 0.239 \\
BEER full & 0.206 & 0.245 & 0.244 & 0.23 & 0.198 & 0.263 & 0.283 & 0.245 & 0.239
\end{tabular}

Table 1: Kendall $\tau$ scores on WMT12 data

\section{PET features}

6. Kendall $\tau$ features

7. all fluency features (PET and Kendall $\tau$ )

Table 1 shows the results sorted by their average Kendall $\tau$ correlation with human judgment.

\section{Analysis}

Given these experimental results, we are coming back to the questions we asked in the introduction.

\subsection{What kind of features are more helpful for sentence level evaluation?}

Fluency vs. Adequacy The fluency features play a smaller role than adequacy features. Apparently, many SMT systems participating in this task have rather similar reordering models, trained on similar data, which makes the fluency features not that discriminative relative to adequacy features. Perhaps in a different application, for example MT system tuning, the reordering features would be far more relevant because ignoring them would basically imply disregarding the importance of the reordering model in MT.

Character vs. Word features We observe that, precision, recall and F-score on character ngrams are crucial. We think that this shows that less sparse features are important for sentence level evaluation. The second best features are word features. Without word features, BEER scores just below METEOR, which suggests that word boundaries play a role as well. In contrast, differentiating between function and content words does not seem to be important.

PETs vs. Kendall $\tau$ Despite the smaller role for reordering features we can make a few observations. Firstly, while PETs and Kendall seem to have similar effect on English-Foreign cases, in all four cases of Foreign-English PETs give better scores. We hypothesize that the quality of the permutations (induced between system output and reference) is better for English than for the other target languages. Discarding PET features has far larger impact than discarding Kendall. Most interestingly, for de-en it makes the difference in outperforming METEOR. In many cases discarding Kendall $\tau$ improves the BEER score, likely because it conflicts with the PET features that are found more effective.

\subsection{Is a linear model sufficient?}

A further insight, from our perspective, is that $F$-score features constitute a crucial set of features, even when the corresponding precision and recall features are included. Because our model merely allows for linear interpolation, whereas F-score is a non-linear function of precision and recall, we think this suggests that a nonlinear interpolation of precision and recall is useful. ${ }^{4}$ By formulating the evaluation as a ranking problem it is relatively easy to "upgrade" for using non-linear models while using the same (or larger) set of features.

\subsection{Train for the language pair or only for the target language?}

All our models were trained for each language pair. This is not the case with many other metrics which train their models for each target language instead of language pair. We contrast these two settings in Table 2. Training for each language pair separately does not give significant improvement over training for the target language only. A possible reason could be that by training for the target language we have more training data (in this case four times more).

\begin{tabular}{l|llll|l} 
Train for & cs-en & fr-en & de-en & es-en & avg $\tau$ \\
\hline target lang & 0.199 & 0.257 & 0.273 & 0.248 & 0.244 \\
lang pair & 0.198 & 0.263 & 0.283 & 0.245 & 0.247
\end{tabular}

Table 2: Kendall $\tau$ scores on WMT12 for different training data

\subsection{BEER vs. METEOR}

The results across individual language pairs are mostly consistent with the averages with a few exceptions. BEER outperforms METEOR in five out of eight language pairs, ties at one (the difference is only 0.001 on es-en) and loses in two (en-fr and cs-en). In some cases BEER is better than METEOR by a large margin (see, e.g., en-cs, en-de).

\footnotetext{
${ }^{4}$ Interestingly, METEOR tunes $\beta$ in $\mathrm{F}_{\beta}$.
} 


\section{Conclusion}

In this work we show that combining less sparse features at the sentence level into a linear model that is trained on ranking we can obtain state-of-the-art results. The analysis of the results shows that features on character ngrams are crucial, besides the standard word level features. The reordering features, while rather important, are less effective within this WMT task, albeit the more abstract PET features have larger impact than the often used Kendall. Good performance of Fscore features leads to the conclusion that linear models might not be sufficient for modeling human sentence level ranking and to learn the right relation between precision and recall it could be worthwhile exploring non-linear models.

\section{Acknowledgments}

This work is supported by STW grant nr. 12271 and NWO VICI grant nr. 277-89-002. We also thank TAUS and the other DatAptor project User Board members.

\section{References}

Alexandra Birch and Miles Osborne. 2010. LRscore for Evaluating Lexical and Reordering Quality in MT. In Proceedings of the Joint Fifth Workshop on Statistical Machine Translation and MetricsMATR, pages 327-332, Uppsala, Sweden, July. Association for Computational Linguistics.

Chris Callison-Burch, Philipp Koehn, Christof Monz, Matt Post, Radu Soricut, and Lucia Specia. 2012. Findings of the 2012 Workshop on Statistical Machine Translation. In Proceedings of the Seventh Workshop on Statistical Machine Translation, pages 10-51, Montréal, Canada, June. Association for Computational Linguistics.

Mark Hall, Eibe Frank, Geoffrey Holmes, Bernhard Pfahringer, Peter Reutemann, and Ian H. Witten. 2009. The WEKA Data Mining Software: An Update. SIGKDD Explor. Newsl., 11(1):10-18, November.

Ralf Herbrich, Thore Graepel, and Klaus Obermayer. 1999. Support Vector Learning for Ordinal Regression. In In International Conference on Artificial Neural Networks, pages 97-102.

Mark Hopkins and Jonathan May. 2011. Tuning as Ranking. In Proceedings of the 2011 Conference on Empirical Methods in Natural Language Processing, pages 1352-1362, Edinburgh, Scotland, UK., July. Association for Computational Linguistics.

Hideki Isozaki, Tsutomu Hirao, Kevin Duh, Katsuhito Sudoh, and Hajime Tsukada. 2010. Automatic Evaluation of Translation Quality for Distant Language Pairs. In Proceedings of the 2010 Conference on Empirical Methods in Natural Language Processing, EMNLP'10, pages 944-952, Stroudsburg, PA, USA. Association for Computational Linguistics.
Hang Li. 2011. Learning to Rank for Information Retrieval and Natural Language Processing. Synthesis Lectures on Human Language Technologies. Morgan \& Claypool Publishers.

Gideon Maillette de Buy Wenniger and Khalil Sima'an. 2011. Hierarchical Translation Equivalence over Word Alignments. In ILLC Prepublication Series, PP-2011-38. University of Amsterdam.

Michael Denkowski and Alon Lavie. 2014. Meteor Universal: Language Specific Translation Evaluation for Any Target Language. In Proceedings of the ACL 2014 Workshop on Statistical Machine Translation.

Markos Mylonakis and Khalil Sima'an. 2008. Phrase Translation Probabilities with ITG Priors and Smoothing as Learning Objective. In Proceedings of the 2008 Conference on Empirical Methods in Natural Language Processing, pages 630-639, Honolulu, USA, October. Association for Computational Linguistics.

Kishore Papineni, Salim Roukos, Todd Ward, and WeiJing Zhu. 2002. BLEU: A Method for Automatic Evaluation of Machine Translation. In Proceedings of the 40th Annual Meeting on Association for Computational Linguistics, ACL '02, pages 311-318, Stroudsburg, PA, USA. Association for Computational Linguistics.

Miloš Stanojević and Khalil Sima'an. 2014. BEER: BEtter Evaluation as Ranking. In Proceedings of the Ninth Workshop on Statistical Machine Translation, pages 414-419, Baltimore, Maryland, USA, June. Association for Computational Linguistics.

Dekai Wu. 1997. Stochastic inversion transduction grammars and bilingual parsing of parallel corpora. Computational linguistics, 23(3):377-403.

Muyun Yang, Junguo Zhu, Sheng Li, and Tiejun Zhao. 2013. Fusion of Word and Letter Based Metrics for Automatic MT Evaluation. In Proceedings of the Twenty-Third International Joint Conference on Artificial Intelligence, IJCAI'13, pages 2204-2210. AAAI Press.

Yang Ye, Ming Zhou, and Chin-Yew Lin. 2007. Sentence Level Machine Translation Evaluation As a Ranking Problem: One Step Aside from BLEU. In Proceedings of the Second Workshop on Statistical Machine Translation, StatMT '07, pages 240-247, Stroudsburg, PA, USA. Association for Computational Linguistics.

Hao Zhang and Daniel Gildea. 2007. Factorization of synchronous context-free grammars in linear time. In In NAACL Workshop on Syntax and Structure in Statistical Translation (SSST.

Hao Zhang, Daniel Gildea, and David Chiang. 2008. Extracting Synchronous Grammar Rules From Word-Level Alignments in Linear Time. In Proceedings of the 22nd International Conference on Computational Linguistics (COLING-08), pages 1081-1088, Manchester, UK. 